\title{
Survey Paper on Sclera Segmentations Techniques
}

\author{
Snehal Rajole ${ }^{1}$, Prof J. V. Shinde ${ }^{2}$ \\ Student, Department of Computer Engineering, K.G.N Sapkal College, Nasik, India ${ }^{1}$ \\ Professor, Department of Computer Engineering, K.G.N Sapkal College, Nasik, India ${ }^{2}$
}

\begin{abstract}
Due to extensive growth of internet, security of the internet is area of interest for the researchers. An efficient biometric trait these days are sclera blood vessels. Sclera is a white region in the eye, around the eyeball which contains blood vessel patterns that can be used for personal identification. In this paper we present the existing sclera segmentation methods and their working in with their limitations and ideas to enhance them.
\end{abstract}

Keywords: Sclera, Segmentation, Iris, Feature extraction, Classificiation.

\section{INTRODUCTION}

Sclera segmentation has gain substantial importance for eye \& iris biometrics. However, sclera segmentation has not been extensively researched as a separate topic, but mainly summarized as a component of a broader task[1]. The sclera is the white and opaque areas of blood vessels and connective tissue within the eye. This part of the eye surrounds the iris which is the colored tissue around the pupil [2].The sclera as shown in Fig. 1, has a rich pattern of blood vessels which have different orientations and layers. Therefore, the discriminant characteristics of these blood vessels are thought a bright factor for eye recognition under visible wavelength illumination [3].
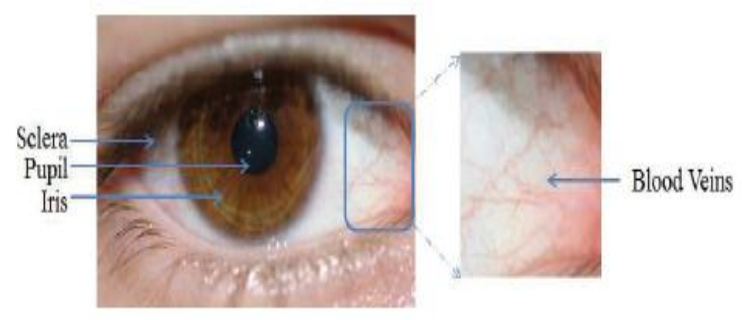

Fig1.Scelera Image

TABLE I Comparison of Biometric Based techniques [3]

\begin{tabular}{|c|c|c|c|c|c|c|c|}
\hline & Accuracy & Reliability & Stable & ID & $\begin{array}{c}\text { ID in } \\
\text { distance }\end{array}$ & $\begin{array}{l}\text { User } \\
\text { Co-op }\end{array}$ & $\begin{array}{c}\text { Large } \\
\text { Population }\end{array}$ \\
\hline Fingerprint & High & Very high & Yes & Yes & No & Yes & Yes \\
\hline Face & Medium & Medium & No & somewhat & somewhat & No & No \\
\hline Iris & Very high & Very high & Yes & Yes & somewhat & Yes & Yes \\
\hline Voice & Low & Low & No & No & - & No & No \\
\hline $\begin{array}{c}\text { Hand } \\
\text { geometry }\end{array}$ & Low & Low & Yes $^{1}$ & No & - & Yes & No \\
\hline Ear shape & Medium & Medium & Yes $^{1}$ & No & somewhat & Yes & No \\
\hline Signature & Low & Low & No & No & - & Yes & No \\
\hline
\end{tabular}

'Patterns remain stable throughout adulthood in normal situations.
In Table I we have shown the comparison of the different biometrics based on the following parameters: accuracy [4], stability [5], reliability [6], [7], identification (ID) and ID capability in a distance [8], user cooperation [6], and scalability to a large population [9].

From this table we can see that Iris has a large scope for research. Sclera can be acquired at a distance under visible wavelength illumination.

Sclera recognition has received attention recently due to the distinctive features extracted from blood vessels within the sclera. However, errant human pose, multiple iris gaze directions, completely different eye image capturing distance and variation in lighting conditions cause several challenges in sclera recognition.

The various challenges in sclera recognition comprises of exact segmentation of the sclera area, sclera vessel enhancement \& the extraction of judicial features of the sclera vessel pattern for authentication \& recognition purposes. The task becomes harder as often a complete sclera image is not received but is impeded by portions of the eyelid \& eyelashes. Moreover, different lighting conditions can change the appearance of the texture patterns by accentuating $\&$ attenuating various grey tones. Also the authentication system should work in real-time so that extraction, representation and comparison of texture images should not consume large computational resources. After that, a classification system uses the mathematical model of the sclera texture to compare with other sclera images to identify specific individuals or recognise an individual.

This paper is organized as follows. Section II covers the background of sclera and sclera segmentation techniques. In Section III we gives the limitations of existing systems and how the advancement can be made in those system. And conclusion is drawn in section IV. 
Vol. 5, Issue 12, December 2016

\section{LITERATURE SURVEY}

Derakhshani et al. [10], the sclera region was manually segmented. Their research studied the feasibility of using blood veins in the sclera as a method for recognition. After that, Derakhshani and Ross [11] investigated a new method for representing and matching the texture of blood vessels using wavelet-derived features and neural network classifiers. In [12], a semi-automated sclera segmentation scheme was used along with an image enhancement and registration scheme to process information in the blood veins of the sclera. Thomas et al. [13] suggested a new automated method for sclera segmentation based on a single skinbased segmentation in the RGB color space. On the other hand, in [14], features describing the blood vessels in the sclera were extracted with Local Binary Patterns (LBPs).

Zhou et al. [15] used bank of Gabor filters with a line descriptor to create a binary blood vessels skeleton map. A discrete Meyer wavelet filter banks and Local Directional Pattern (LDP) were used in [16] for blood vessel enhancement and feature extraction. Finally, Alkassar et al. [17] proposed a new sclera segmentation and occluded eye detection for sclera validation.

One of the first papers that describes sclera segmentation employs a modified Self Organizing Map [18] in a gaze tracking approach. The method depends on discovering the iris boundary first \& fixing two control positions estimated by using iris center and radius. The two control positions are then employed in an active contour model algorithm to fine tune the sclera boundary location. In [19] it is suggested that sclera recognition should be done only on the sclera vein patterns layer, which are stable over time, rather than including the conjunctiva vasculature. The sclera segmentation approach employed in [19] assumes that the images contain frontal-looking eyes and the iris centre location is available. Two binary maps are produced based on observing non-skin area using RBG colour space \& white colour using HSV colour space. Furthermore, the convex hull of the two masks is calculated and mixed to gain a final sclera region.

In[20] the authors explored Sclera vasculature as a biometric modality under various wavelengths. The sclera was segmented by employing a sclera index measure, which relies on multispectral information, i.e. the difference between near infrared and green pixel intensities is larger for the sclera region. In [21] a K-means clustering approach is employed to segment the sclera. A survey of the sclera recognition works until 2013 was made in [22] and with regards to sclera segmentation the survey shows that the few existing approaches are relying on various assumptions, e.g. iris centre location is known. In 2014, Abhijit et al proposed a method for sclera segmentation based on Fuzzy logic [23].

A typical sclera biometric system is explained in Fig. 2.

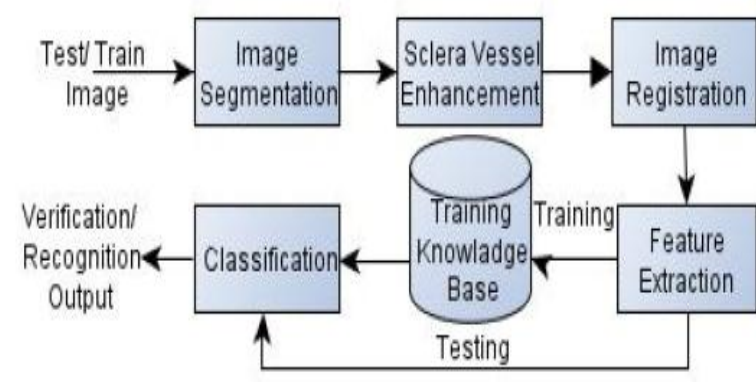

Fig2. Typical Sclera Biometric system

A. Sclera Segmentation

Segmentation is the first step for most biometric related research. Here the main aim is to identify the region of interest as appropriate as possible. Similarly in sclera biometric a perfect segmentation is important otherwise, an incorrect segmentation can reduce the pattern available, but also it can introduce other patterns such as eyelashes and eyelids. So in the literature of sclera biometric, the researchers have given a great importance to this phase.

Many binary skin-based segmentation algorithms have been developed to segment skin area on a human body depending on a different color space such as RGB, HSV, HIS and $\mathrm{YCbCr}[24,25]$. The performance of these methods have achieved satisfactory results in terms of accuracy and complexity. However, these methods were tested only on limited databases with some having constrained imaging conditions. In addition, recent pixelbased sclera segmentation methods have used one color space which is not robust against noise factors and unconstrained imaging necessitating more post-processing complexity. As a result, sclera segmentation design depending on multiple color spaces will ensure the versatility of segmentation for constrained and unconstrained eye imaging but the complexity could be considerable.

Possibly [26] was the first work on automated sclera segmentation. Here, the sclera was segmented by a timeadaptive active contour-based method. The iris was localized in the detected eye strip in the binary image through template matching via an adaptive half-circle template.

A TASOM (Time Adaptive Self-Organizing Map)-based active contour method detailed in [27], [28] was used to get the inner boundary of the sclera. In [29], the authors have designed enhancement and registration methods to process and match conjunctival vasculature obtained under non-ideal conditions.

In [30], a colour image sclera segmentation process was proposed, which includes image down sampling, conversion to the HSV colour space, estimation of the sclera region, iris and eyelid detection, eyelid and iris boundary refinement, mask creation, and mask upsampling. In [31], a robust multi-angled sclera recognition technique was proposed. A new robust method of sclera segmentation for colour images was proposed in [32]. 
Vol. 5, Issue 12, December 2016

B. Sclera vessel enhancement and Image registration The vessels in the sclera are not prominent, so in order to make them clearly visible, image enhancement is required. Various techniques for sclera vessel enhancement are found in literature. The first recognized work on sclera vessels enhancement is recorded in [33]. In [29] for image registration a local affine and a global smooth transformation was applied. A image mapping method was used in [34] to make the images invariant to rotation. In [35] and [30] sclera vain pattern enhancement by Gabor filter. In [36] selective enhancement filter for lines, and implicitly for blood vessels, as described in [37] and used in [38] was applied to the green component.

\section{Feature extraction}

The feature extraction of sclera recognition system involves in building a reliable mathematical model of the abstract sclera pattern to reliably identify persons for authentication and identification purposes.

In [39] the Discrete Cosine Transform and wavelets were used for feature representation. [40] GLCM (Grey Level Cooccurrence Matrix) was used for sclera biometrics. The authors in [31] presented four fusion methods for combining recognition results from multi-angle images. LBP (Local Binary Pattern) feature was used for sclera biometrics in [41]. A tile-based feature extraction method was used for sclera biometrics in [34].

\section{Classification}

Biometric algorithms generally aim to provide a reasonable answer for all possible inputs so classification plays a important role, its importance is also reflected in sclera literature. In [33] classification was performed by template matching against the stored template for verification by one-to-one matching was used.

For classification, template matching was used in [31]. In [32], [35] for classification, the Hamming distance was used for template-based matching. In [31] after feature extraction and matching technique SURF (Speeded Up Robust Features) was used for key point matching.

A feed forward Neural Network with a single hidden layer was used here for classification in [39]. In [40] match score level fusion of Fisher LDA and neural networks are used which provides the best results in classification.

\section{III.DISCUSSION}

The literature relevant to sclera biometrics is not large, but is growing rapidly and spreads across a wide variety of sources. This survey suggests a structure for the sclera biometrics literature and summarizes the current state-oftheart. There are still a number of active research topics within Sclera biometrics. Many of these are related to the desire to make sclera recognition practical in lesscontrolled conditions and also a real time process as much as possible.
Limitations and challenges remain. These are: 1) sclera segmentation has not been investigated using heavily noise eye images; 2) Research in eye rotation alignment method is still missing which could affect the blood vessel angles and position matching; 3) sclera recognition has not been extensively investigated when eye images are captured onthe-move and at-a-distance.

\section{IV.CONCLUSION}

In this paper we studied the existing sclera segmentations methods which are use for different purposes, Biometric trait is one of the major applications for the sclera segmentation. The existing systems and their disadvantages are shown in section 2 and 3 . In future the sclera methods can be enhanced by using eye gaze detection technique.

\section{ACKNOWLEDGMENT}

My sincere thanks go to KCTs Late G N Sapkal college of Engineering for providing strong platform to develop skills and capabilities. I could never have completed paper without the support and assistance of many people. First and foremost, I would like to express deepest gratitude to my guide Prof. N. R. Wankhade for his excellent guidance, valuable suggestions and kind of encouragement in academics.

\section{REFERENCES}

[1] S. Alkassar, W. L. Woo, S. S. Dlay and J. A. Chambers, "A novel method for sclera recognition with images captured on-the-move and at-a-distance," 2016 4th International Conference on Biometrics and Forensics (IWBF), Limassol, 2016, pp. 1-6.

[2] C. Oyster, The Human Eye: Structure And Function. Sinauer Associates Incorporated, 1999. [Online]. Available: http://books.google.co.uk/books?id=n9yoJQAACAAJ, accessed

[3] Z. Zhou, E. Y. Du, N. L. Thomas and E. J. Delp, "A New Human Identification Method: Sclera Recognition," in IEEE Transactions on Systems, Man, and Cybernetics - Part A: Systems and Humans, vol. 42, no. 3, pp. 571-583, May 2012.

[4] Y. Du, "Biometrics: Technologies and Trend," in Encyclopedia of OpticalEngineering. New York: Marcel Dekker, 2006.

[5] Y. Du, "Biometrics," in Handbook of Digital Human Modeling. Mahwah, NJ: Lawrence Erlbaurm, 2008.

[6] K. Mahadevan, "Estimating Reliability Impact of Biometric Devices in Large Scale Applications," M.S. thesis, West Virginia Univ.,Morgantown, WV, 2003.

[7] A. K. Jain, A. Ross, and S. Prabhakar, "An introduction to biometric recognition," IEEE Trans. Circuits Syst. Video Technol., vol. 14, no. 1, pp. 4-20, Jan. 2004.

[8] S. Crihalmeanu, A. Ross, S. Schuckers, and L. A. Hornak, "A Protocol for Multibiometric Data Acquisition, Storage and Dissemination," WVU, Lane Dept. Comput. Sci. Elect. Eng., Morgantown, WV, Tech. Rep.,2007.

[9] A. K. Jain, A. Ross, and S. Pankanti, "Biometrics: A tool for information security," IEEE Trans. Inf. Forensics Security, vol. 1, no. 2, pp. 125-143, Jun. 2006.

[10] R. Derakhshani, A. Ross, and S. Crihalmeanu. A new biometric modality based on conjunctival vasculature. Proc. Of Artificial Neural Networks in Engineering (ANNIE), pages 1-8, 2006.

[11] R. Derakhshani and A. Ross. A texture-based neural network classifier for biometric identification using ocular surface vasculature. In Int. Joint Conf. on Neural Networks. IJCNN., pages 2982-2987. IEEE, 2007. 
Vol. 5, Issue 12, December 2016

[12] S. Crihalmeanu and R. Derakhshani. Enhancement and registration schemes for matching conjunctival vasculature. In Proc. of the 3rd IAPR/IEEE International Conference on Biometrics (ICB, pages 1240-1249, 2009.

[13] N. L. Thomas, Y. Du, and Z. Zhou. A new approach for sclera vein recognition. In SPIE Defense, Security, and Sensing, pages 770805-770805. International Society for Optics and Photonics, 2010.

[14] K. Oh and K.-A. Toh. Extracting sclera features for cancelable identity verification. In Biometrics (ICB), $20125^{\text {th }}$ IAPR International Conference on, pages 245-250, 2012

[15] Z. Zhou, E. Y. Du, N. L. Thomas, and E. J. Delp. A new human identification method: sclera recognition. 42(3):571 583, 2012.

[16] A. Das, U. Pal, M. A. F. Ballester, and M. Blumenstein. A new efficient and adaptive sclera recognition system. In IEEE Symp. on Computational Intelligence in Biometrics and Identity Management (CIBIM), pages 1-8. IEEE, 2014.

[17] S. Alkassar, W. L. Woo, S. S. Dlay, and J. A. Chambers. Robust sclera recognition system with novel sclera segmentation and validation techniques. IEEE Transactions on Systems, Man, and Cybernetics: Systems, PP(99):1-13, 2016.

[18] M. H. Khosravi and R. Safabakhsh. Human eye sclera detection and tracking using a modified time-adaptive self-organizing map. Pattern Rec., 41(8): 2571-2593, 2008.

[19] Z. Zhi, E. Y. Du, N. L. Thomas, and E. J. Delp. A New Human Identification Method: Sclera Recognition. IEEE Trans. Syst., Man and Cyb., Part A, 42: 571-583, 2012.

[20] S. Crihalmeanu and A. Ross. Multispectral scleral patterns for ocular biometric recognition. Pattern Recognition Letters, 33:18601869, 2012.

[21] S. Crihalmeanu, A. Ross, and R. Derakhshani. Enhancement and Registration Schemes for Matching Conjunctival Vasculature. Adv. in Biometrics. 5558: 1240-1249, 2009.

[22] A. Das, U. Pal, M. Blumenstein, and M. A. Ferrer Ballester. Sclera Recognition - A Survey. 2nd IAPR Asian Conf. on Pattern Rec. (ACPR): 917-921, 2013.

[23] A. Das, U. Pal, M. A. Ferrer Ballester and M. Blumenstein. A new efficient and adaptive sclera recognition system. Computational Intellig. in Biometrics and Identity Management, IEEE Symposium on, 2014.

[24] D. Chai and K. N. Ngan. Face segmentation using skincolor map in videophone applications. Circuits and Systems for Video Technology, IEEE Transactions on, 9(4):551-564, 1999.

[25] C. Garcia and G. Tziritas. Face detection using quantized skin color regions merging and wavelet packet analysis. Multimedia, IEEE Transactions on, 1(3):264-277, 1999.

[26] M. H. Khosravi and R. Safabakhsh, Human eye sclera detection and tracking using a modified time-adaptive self-organizing map, Pattern Recognition, vol. 41, pp 2571 -2593, 2008.

[27] H. Shah-Hosseini, R. Safabakhsh, TASOM: a new time adaptive self organizing map, IEEE Trans. Syst. Man Cybern. Part B, vol. 33 ,no. 2,pp. 271-282, 2003.

[28] H. Shah-Hosseini, R. Safabakhsh, A TASOM-based algorithm for active contour modelling, Pattern Recognition Letter, vol. 24 , no. 9, pp. 1361-1373 2003.

[29] S. Crihalmeanu , A. Ross, and R. Derakhshani, Enhancement and Registration Schemes for Matching Conjunctival Vasculature, Appeared in Procceding of the 3rd IAPR/IEEE International Conference on Biometrics, pp.1247-1256, 2009

[30] Z. Zhou, E. Y. Du, and N. L. Thomas, A Comprehensive Sclera Image Quality Measure, 11th International Conference on Control, Automation, Robotics and Vision, pp. 638-643, 2010.

[31] Z. Zhou, Y. Du, N. L. Thomas, and E. J. Delp, Multi-angle Sclera Recognition System, IEEE Workshop on Computational Intelligence in Biometrics and Identity Management: pp. 103 -108, 2011.

[32] Z. Zhou, Y. Du, N. L. Thomas, and E. J. Delp, A New Human Identification Method: Sclera Recognition, IEEE transaction on System, Man And Cybernatics PART A: System And Human, vol. 42, no. 3, pp - 571-583, 2012.

[33] R. Derakhshani, A. Ross, and S. Crihalmeanu, A new biometric modality based on conjunctival vasculature. Proceedings of Artificial Neural Networks in Engineering, pp. 1-8, 2006.
[34] V. Gottemukkula, S. K. Saripalle, S. P. Tankasala, R. Derakhshani, R. Pasula and A. Ross, Fusing Iris and Conjunctival Vasculature: Ocular Biometrics in the Visible Spectrum, IEEE Conference on Technologies for Homeland Security, pp. 150-155, 2012.

[35] Z. Zhou, Y. Du, N. L. Thomas, and E. J. Delp, Multimodal eye recognition. Proc. of the International Society for Optical Engineering, vol.7708, no. 770806, pp. 1-10, 2010.

[36] S. Crihalmeanu and A. Ross, Multispectral sclera patterns for ocular biometric recognition, Pattern Recognition Letters, vol 33, pp. 1860- 1869, 2012.

[37] L. Qiang, , S. Shusuke, D. Kunio, Selective enhancement filters for nodules, vessels, and airway walls in two or three dimentional CT scans. Medical. Physics. vol.30, no. 8, pp. 2040-2051, 2003.

[38] S. Crihalmeanu, A. Ross,. On the use of multispectral conjunctival vasculature as a soft biometric. In: Proc. IEEE Workshop on Applications of Computer Vision (WACV), pp. 204-211, 2011.

[39] R. Derakhshani and A. Ross, A texture-based neural network classifier for biometric identification using ocular surface vasculature, Proc. Of International Joint Conference on Neural Networks, pp. 2982-2987, 2007.

[40] S. P. Tankasala, P. Doynov, R. R. Derakhshani, A. Ross and S. Crihalmeanu, Biometric Recognition of Conjunctival Vasculature using GLCM Features, International Conference on Image Information Processing, pp. 1-6, 2011

[41] K. Oh and K. Toh, Extracting Sclera Features for Cancelable Identity Verification, 5th IAPR International Conference on Biometric, pp. 245-250, 2012.

\section{BIOGRAPHY}

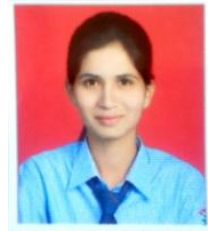

Snehal S. Rajole completed her graduation from K.V.N. Naik college of Engineering Nashik, Maharashtra presently she is perusing her post graduation from L.G.N. Sapkal college of Engineering Nashik, Maharashtra India. Her research of interest include image processing, Sclera segmentation, Sclera recognition, pattern recognition. 